\title{
A Study on the Garden Path Phenomenon from the Perspective of Generative Grammar
}

\author{
Haojie Li \\ School of Foreign Languages, Southwest University of Political Science and Law, Chongqing, China \\ Xiangkun Sheng \\ Marine and Fishery Supervision Brigade of Laizhou, Yantai, Shandong, China
}

\begin{abstract}
Studies in the past mainly focus on the garden path phenomenon from the perspective of cognitive linguistics and psycholinguistics. The adequacies of their explanations are different, but these theories are imperfect. This paper discusses the garden path phenomenon from the perspective of generative grammar. The author holds that the $\boldsymbol{\theta}$-attachment principle can analyze the reason of partial ambiguity of the garden path phenomenon effectively and the garden path phenomenon provides evidence for derivation by phase under the framework of MP in generative grammar. People can clarify the structure of the garden path phenomenon succinctly and enhance the understanding of language mechanism through the analysis of them from the perspective of generative grammar. The faculty of language, which is the biological object, must abide by organism operation law as well. The memories of human beings are limited and cannot load too many syntactic structures at one time. As a result, the faculty of language can simply deal with a limited structure at a time. Only the limited amount of structural information can be accommodated in the active memory and phased spelling can reduce the memory load.
\end{abstract}

Index Terms - the garden path phenomenon, generative grammar, psycholinguists, partial ambiguity, the active memory, $\theta$-attachment principle, derivation by phase

\section{INTRODUCTION}

The garden path phenomenon is a temporary partial ambiguity in the process of language parsing, which is kept a watchful eye on by linguists and psycholinguists. This special phenomenon was noticed, discussed and researched centering on its properties and characteristics in around the nineteen seventies. Garden path sentence refers to sentences in (1).

(1) a. The horse raced past the barn fell.

b. The girl told the stories cried.

c. The senator criticized on radio resigned.

d. The raft floated down the river sank.

e. Fat people eat accumulates.

f. The old man the boat.

(1) a is a typical Garden Path sentence, which is easily understood as a structure of NP +V + PP by the readers. But the readers will realize that a processing mistake occurs when they see the word "fell". Then they parse the whole sentence again and re-process it. Feng Zhiwei and Xu Fuji (2003) makes a vivid description of Garden Path Phenomenon: Just as we walked into a picturesque garden, looking for the exit of the garden, most people thought that the exit must be at the end of the main path of the garden, so that it could walk along the main path naturally and leisurely towards the garden. Just as we walked along the main path of the garden to enjoy the beauty of the garden and suddenly found that this path was wrong, it did not lead to the exit of the garden, and the right path to the exit was the other one next to the main path and it is almost forgotten by the visitors.

Other scholars also give the definition of Garden Path Phenomenon, such as Carroll (2000), Hu (2001) and Jay (2004).

Carroll (2000) argues that we interpret a sentence in a particular way only to find out near the end that we misinterpreted it. The subjective impression is that of being led down a garden path until discovering at the end we took the wrong path and have to retrace our efforts. It is called the garden path effect.

$\mathrm{Hu}$ (2001) holds that garden path sentences are sentences that are initially interpreted with a different structure than they actually have.

Jay (2004) thinks that a garden path sentence is a metaphor for being led down a linguistic path that takes us to the wrong meaning.

Based on generative grammar, this paper aims to analyze the cause of Garden Path Phenomenon and discuss that this phenomenon provides an evidence to Phase Theory. The paper consists of four chapters. After the introduction in Chapter 1, the second chapter is about the literature review: previous research findings by researchers home and abroad 
as well as their deficiencies. Analysis and discussions are implemented in the third chapter. The final chapter serves as the conclusion of the paper.

\section{LiterATURE REVIEW}

Many studies have been carried out regarding Garden Path Phenomenon since Bever (1970) first come up with this phenomenon. These studies, however, mainly focus on garden path phenomenon from the perspective of cognitive linguistics and psycholinguistics. The adequacies of their explanations are different, but these theories are imperfect.

\section{A. Bever's Hierarchy of Canonical Schemas}

Bever (1970) put forward a hierarchy of canonical schemas, which maintains listeners check in turn the language input and the brain's various leveled standard schemas in the language processing. If a certain language input matches the highest level of the schema, it is accepted and processed; if not, it needs to find a corresponding lower-level schema to determine the sentence structure of the language input. Take (1) b as an example. In fact, "told the stories" is equivalent to a restrictive attributive clause in nature, which omits "who was" that modifies the subject "the girl". However, listeners tend to correspond "the girl told the stories" to a high level standard schema "actor ... action ... modifier" in the brain and understand it as a sentence structure when they do not encounter the word "cried". When the word "cried" appears, listeners suddenly realize that there is an error in the process of language processing and then are forced to turn back to re-analyze and deal with the language processing, resulting in the difficulties of language processing and understanding. As a result, "garden path phenomenon" comes into being.

\section{B. Kimball's Seven Principles of Syntactic Analysis}

Kimball $(1973,1975)$ holds that syntactic analysis is divided into two levels, the first level analyzes the sentence component structure of the surface structure successively and then sends them to the second level for deep processing part by part. Kimball proposes seven principles of syntactic analysis: a. the principle of two sentences b. the principle of right association $c$. the principle of early closure $d$. the principle of fixed structure e. the top-down principle $f$. the principle of new nodes g. the principle of processing. Among them, a-c are regarded as the tools to explain the reasons of ambiguity of garden path phenomenon. Take (2) and (3) for example.

(2) a. The other boy the girl the boy liked loved went abroad.

b. The boy liked the girl that loved the other boy that went abroad.

In the two sentences, (2)a is harder to understand than (2)b because the embedded clause is added to interrupt people's understanding in (2)a. As a result, (2)a violates the principle of two sentences and it is unintelligible. In general, the brain's direct memory can simply accommodate two independent clauses or sentences syntactically. This can explain why embedded clauses that are added to sentences will interrupt the people's understanding process.

(3) [John believes [that Mary left]] is clear.

The processing of (3) is subject to both the principle of right association and the principle of early closure. The processing of (3) confirms to the principle of right association as shown in the brackets above. When the components outside the brackets appear, according to the principle of early closure, the existing components have become an integral phrase and should be closed. Therefore the new components are excluded. To incorporate the new components into the existing structure of the sentence, it is necessary to break the structure of the sentence that has been established and to re-analyze it syntactically, resulting in difficulties in language processing.

\section{The Sausage Machine of Frazier \& Fodor}

Frazier \& Fodor (1978) put forward the language processing strategies in order to explain the garden path phenomenon, including late closure strategy and minimal attachment principle.

Late closure strategy stipulates, if possible, people attach new items to the current components and its main basis is that this strategy can reduce the burden of working memory. Take (4) for example.

(4) Tom said that Bill had taken the cleaning out yesterday.

In (4), the adverb "yesterday" can either be attached to the main clause "Tom said..." or be attached to the subordinate clause "Bill had taken...", but people generally take the latter. Frazier\& Fodor investigate the fixation vision of the subjects in reading the structurally ambiguous sentence (5).

(5) Since Jay always jogs a mile seems like a very short distance to him.

To some extent, the ambiguity of (5) is made on purpose because it lacks a comma after the word "jogs". But Frazier $\&$ Fodor's discovery is quite interesting because the time of the fixation vision of the subjects to the former words is longer than the latter. This shows that the subjects have some misunderstandings to the phrase "a mile" and some revisions should be made.

Minimal attachment principle can also be called local attachment strategy to compensate for the deficiency of the principle of right association. According to this rule, people tend to connect new items to phrase markers that use the least nodes. Take (6) as an example.

(6) John kissed Mary and her sister.

There are two explanations for sentence (6), one is the noun phrase that is connected with a conjunction: "Mary and 
her sister", and the other is the conjunction followed by the noun as the beginning of another noun phrase: "and her sister". In accordance with the "minimal attachment principle", people tend to take the previous explanation: "Mary and her sister", because it uses fewer syntax nodes. More examples come from (7).

(7) a. The city council argued the mayor's position forcefully.

b. The city council argued the mayor's position was incorrect.

(7) a conforms to minimal attachment principle because the adverb "forcefully" is connected to the current verb phrase component "argued the mayor's position". In contrast, (7) b is a complementary structure that "argued the mayor's position" requires the creation of a new component.

To sum up, we can see that the garden path sentences have three characteristics: a. the garden path sentences are the temporary local ambiguous sentences, the front part of the sentence is ambiguous and the whole sentence is not ambiguous. b. There is a priority between different ambiguity results in the process of understanding the front ambiguous of the garden path sentence. Some ambiguous explanations are what people are willing to accept, and some are what people are unwilling to accept. c. The explanation that people do not want to accept is precisely the correct analysis of the garden path sentence (Feng, 2003a: 340-341).

\section{ANALYSIS AND DiSCUSSIONS}

Up to know, focuses on the garden path phenomenon mainly relate to cognitive linguistics and psycholinguistics. Next we intend to make a tentative study the garden path phenomenon from the perspective of generative grammar. We will use theta-attachment (also $\theta$-attachment) principle to analyze the reason of partial ambiguity of the garden path phenomenon and then we will illustrate that the garden path phenomenon provides evidence for derivation by phase.

\section{A. Theta-attachment Principle and the Garden Path Phenomenon}

Pritchett $(1988,1992)$ uses $\theta$-attachment principle, which is derived from government and binding theory, to parse the reason of partial ambiguity of the garden path phenomenon. He thinks that the emergence of the garden path phenomenon is due to the violation of $\theta$-attachment principle when people deal with local ambiguous structures.

(8) $\theta$-attachment principle: The $\theta$-criterion attempts to apply at every point during processing given the maximal $\theta$-grid.

In (8), $\theta$-grid refers to the semantic grid, which can confirm the semantic role group to accept the $\theta$-roles, such as agent, patient, theme and the like. The $\theta$-roles are mainly distributed by verbs or prepositions (Chomsky 1981, 1986; Pritchett 1988).

$\theta$-attachment principle can determine the attachment relationship during the language processing. Once the $\theta$-role to be assigned appears, it must be managed to assign it to a target that can be found to accept the $\theta$-role. As a result, in the process of syntactic reanalysis, the analyst continues to recall that the $\theta$-marked syntactic elements are moved from their current range of $\theta$, resulting in difficulties in language processing and then leading to the garden path phenomenon. Sentences (9) and (10) illustrate the use of $\theta$-attachment principle (Pritchett, 1988).

(9) Without her contributions we failed.

The word "without" appears first and is recognized as a preposition, it can be assigned a $\theta$-role. However, there is not any potential role to accept this $\theta$-role at that time. Then the word "her" appears and it is recognized as an NP. Since there is a $\theta$-role to be assigned for the word "without", and the word "her" as an NP is the potential target that can accept the $\theta$-role. According to the $\theta$-attachment principle, "her" should be attached in a structural position that can accept the $\theta$-role, so "her", as the object of a preposition attaches to the word "without". Then there comes the word "contributions", which is identified as an NP and also an target that can accept the $\theta$-role. So, "her" and "contributions" form a larger NP and the word "contributions" is the head, which gets a $\theta$-role from "without" to meet the $\theta$-attachment principle. But when the word "we" appears, there is no $\theta$-role to be assigned, and "we" cannot form a new NP with "her contributions", so it is in a no role situation and violates the $\theta$-attachment principle. However, according to Pritchett's theory, the violation of the $\theta$-attachment principle is in the inevitable state and will not result in any special difficulties in the language processing process. Finally, the word "failed" occurs. As a verb, "failed" has a $\theta$-role to be removed, so it is assigned to the NP "we" and then "we" as the subject of the verb attaches to "failed". Then we will turn to sentence (10). (10) is a garden path sentence because of the violation of the $\theta$-attachment principle during language processing.

(10) Without her contributions failed to come in.

The sentence is treated the same as sentence (9) before the word "failed" appears. But when "failed" occurs, it has a $\theta$-role to be dismounted, and the $\theta$-role to be dismounted must be assigned to a target with the subject status. However, at this time there is no sentence element that can bear the task. So the noun phrase "her contributions" must be re-analyzed and re-processed in order to remove the $\theta$-role of "failed" to meet the $\theta$-attachment principle. This reanalysis has created special difficulties in dealing with the language. Here "failed" has a $\theta$-role to be removed and there is no component to accept it. This situation is quite different from sentence (9), which "we" waits to accept a $\theta$-role in (9). (10) results in language processing difficulties while (9) will not.

\section{B. Derivation by Phase and the Garden Path Phenomenon}

Derivation by phase is put forward in the process of MP development. In recent MP framework, Chomsky (2001, 
2007, 2008) put forth the derivation of syntactic structures is conducted on the basis of the unit of phase because faculty of language, which is the biological object, must abide by organism operation law. The memories of human beings are limited and could not load too many syntactic structures at a time. The faculty of language can simply deal with a limited structure at a time. Only the limited amount of structural information can be accommodated in the active memory. The goal of derivation by phase is to reduce computational burdens and enhance computational efficiency and Chomsky proposes that the derivation of the syntactic structure is composed of one phase after another, which is derived on the basis of phase. Derivation by phase is something like the principle of processing (Kimball, 1973), but it is much more concrete. Kimball (1973) proposes the principle of processing, which holds that a phrase is transferred to a syntactic or semantic processing phase when it is closed and is cleared from the short memory. However, the theory of derivation by phase considers the problem of load of active memory: phased spelling can reduce the memory load. Syntax theory, which considers the memory load, shows that the syntactic scholars realize that the grammar is somewhat constrained by the language performance. In recent MP framework, the basic sentence structure is [CP > $\left.\mathrm{TP}>\mathrm{V}^{*} \mathrm{P}>\mathrm{VP}\right]$ and $\mathrm{CP}$ and $\mathrm{v}^{*} \mathrm{P}$ are phases, which are complete propositional structures. $\mathrm{v}^{*} \mathrm{P}$ possesses complete argument structure and $\mathrm{CP}$ includes tense, event structure and force. Force is the element to represent sentence types. All syntactic operations are determined by the heads of phase $\mathrm{CP}$ and $\mathrm{v} * \mathrm{P}$ and conduct derivations through phase units. In the light of principles of derivation by phase, the formation of sentences is through the combination of two syntactic elements, the order is from below to above, from right to left in linear structures.

The misinterpretation of the garden path sentence just proves the rationality of derivation by phase. We will take (1) a, which is repeated in (11), as an example to illustrate this point.

(11) The horse raced past the barn fell.

(11) is a typical garden path sentence, which will be reanalyzed when it is usually misunderstood. We are used to understand the front part of this sentence "The horse raced past the barn" in accordance with the grammatical structure of NP+VP+PP. However, when listening/seeing the verb "fell", we will be aware of the mistakes of the understanding, and then turn back to re-processing of the language input only to find the word "raced" is not a verb, but a past participle and the whole sentence is a reduced attributive clause: The horse which was raced past the barn fell. The procedure of understanding (11) shows that the front part of the sentence "The horse raced past the barn" is mistaken for the whole sentence. But when "fell" appears, people will reassemble the elements of the sentence. There are at least two points that deserve our attention. One is that people should finish reading the whole sentence in order to get the correct interpretation. The other is that people tend to parse the sentence part by part. Here "part" is exactly the phase in generative grammar. Then we will analyze (11) by using the theory of derivation by phase and illustrate that derivation by phase from below to above is the only right way to avoid partial ambiguity of the garden path phenomenon.

In terms of the principle of from below to above, relevant lexical items should be selected from lexicon and form VP through merging, as shown in (12).

(12) [vp the horse [v fell]

And then the verb "fell" moves to the place of light verb $\mathrm{v}^{*} \mathrm{P}$, "the horse" moves to the specifier of $\mathrm{V}^{*} \mathrm{P}$, "fell" and "the horse" in the original position are erased, and get (13).

(13) $\left[v^{*}\right.$ the horse [ $v^{*}$ fell [vP the horse [v fell]]]

Then the VP "raced past the barn" adjoins "the horse" and get (14).

(14) $\left[{ }^{*} * \mathrm{P}\right.$ [NP the horse [vP raced past the barn] [ $\mathrm{v}^{*}$ fell [vP the horse [v fell]]]]]

Finally, "the horse" moves to specifier of TP and "the horse" is erased at the same time, as illustrated in (15).

[15] [TP the horse $\left[_{\mathrm{T}}\left[\mathrm{v}_{\mathrm{T}}\right.\right.$ [NP the horse [vP raced past the barn] [ $\mathrm{v}^{*}$ fell [vP the horse [v fell]]]]]]

Up to now, the derivation is over and the whole sentence is transferred to the phonetic level to spell out. The partial ambiguity of the garden path phenomenon is solved successfully by derivation by phase.

\section{CONCLUSION}

The garden path phenomenon is a temporary syntactic ambiguous phenomenon, it is generated by people's inappropriate use of methods to deal with it before the non-ambiguous elements are successfully processed. It is an interesting and complex psychological phenomenon, and language psychologists have proposed different syntactic strategies or models to deal with it. These syntactic strategies or models help us understand the mechanisms of the human brain processing and the process of language understanding (Liu\&Liu, 2004). The reason for the emergence of a garden path phenomenon is that we analyze sentences in a way that is added word by word. When we hear a word, we will speculate on how it will affect the meaning of the whole sentence, and before the other parts of the sentence are heard, the judgment of the word will be made. This is a very necessary strategy, based on the limited memory resources and the requirements of the quick understanding of the exchange of information. But this is also a risky strategy, and sometimes we realize that we have made the wrong choice when we hear the rest of the sentence and have to reanalyze it. The garden path phenomenon reflects the pattern of language processing and understanding, which implies that the understanding may be based on the surfaced, and even the seemingly biased representation. The adequacies of their explanations are different, but these theories are imperfect. We take theta-attachment (also $\theta$-attachment) principle to analyze the reason of partial ambiguity of the garden path phenomenon and then we illustrate that the garden path phenomenon provides evidence for derivation by phase. We can avoid the occurrence of such ambiguous sentences in 
the process of language processing effectively and deal with them better by knowing the causes and types of them. We hope that people can clarify the structure of the garden path phenomenon succinctly (Radford, 1997) and enhance the understanding of language mechanism through the analysis of them from the perspective of generative grammar.

\section{ACKNOWLEDGEMENTS}

This research is funded by General Project of 2017 Chongqing Municipal Education Commission Humanities and Social Sciences Research, P.R.C. Project Name: A Generative Study on the Derivation of the Oblique Object Construction in Mandarin Chinese under the Framework of Distributed Morphology (No. 17SKG003) and 2017 Key Project of Southwest University of Political Science and Law, P.R.C. Project Name: A Syntactic Study on the Verb-copying Construction in Mandarin Chinese (No. 2017XZZD-09).

\section{REFERENCES}

[1] Bever, T. G. (1970). The cognitive basis for linguistic structures. In Hayes, J. R. (ed.). Cognition and the Development of Language. New York: Wiley.

[2] Carroll, D. W. (2000). Psychology of language. Beijing: Foreign Language Teaching and Research Press.

[3] Chomsky, N. (1981). Lectures on Government and Binding. Dordrecht: Foris.

[4] Chomsky, N. (1986). Barriers. Cambridge: MIT Press.

[5] Chomsky, N. (2001). Derivation by phase. In M. Kenstowicz (eds.). Ken Hale: A Life in Language. Cambridge, Mass.: MIT Press, 1-52.

[6] Chomsky, N. (2007). Approaching UG from below. In U. Sauerland (ed.). Interfaces+Recursion=Language? Berlin: Mouton de Gruyter, 1-29.

[7] Chomsky, N. (2008). On phases. In R. Freidin., C. Otero. \& M. L. Zubi-zarreta. (eds.) Fouudarioual issues

[8] Feng, Z. W. (2003a). The automatic parsing algorithm for garden-path sentence. Contemporary linguistics 4: 340-341.

[9] Feng, Z. W. \& F. J. Xu. (2003b). A preliminary study on the garden path. Reading the paper at the HNC (2nd) Symposium in May 2003.

[10] Frazier, L. \& J. Fodor. (1978). The sausage machine: A new two-stage parsing model. Cognition 6: 291-325.

[11] Hu, Z. L. (2001). Linguistics: a course book. Beijing: Beijing University Press.

[12] Jay, T. B. (2004). The psychology of language. Beijing: Beijing University Press.

[13] Kimball, J. (1973). Seven principles of surface structure parsing in natural language. Cognition 2: 15-47.

[14] Kimball, J. (ed.). (1975). Syntax and semantics. Volume 4. New York: Academic Press.

[15] Liu, X. Y. \& G. L. Liu. (2004). Psycholinguistics. Beijing: Higher Education Press.

[16] Pritchett, B. L. (1988). Garden path phenomena and the grammatical basis of language processing. Language 64:539-576.

[17] Pritchett, B. L. (1992). Grammatical competence and parsing performance. Chicago: The University of Chicago Press.

[18] Radford, A. (1997). Syntax: a minimalist introduction. Cambridge, England: Cambridge University Press.

Haojie Li was born in Laizhou, Shandong, China in 1974. He received his M.A. degree in Sichuan International Studies University, China in 2005.

$\mathrm{He}$ is currently a lecturer in the School of Foreign Languages, Southwest University of Political Science and Law, Chongqing, China. His research interests include syntax and theoretical linguistics. He has published a number of studies in his areas of expertise, which have appeared in scholarly publications in China and Europe.

Xiangkun Sheng, who was born in Laizhou, Yantai, Shandong, China in 1973, is currently a clerk at Marine and Fishery Supervision Brigade of Laizhou, Yantai, Shandong, China. His research interest is law English. 